\title{
Mapeamento sistemático acerca das práticas docentes com o uso de Recursos Educacionais Abertos
}

\author{
Emmerson Oseildo Fernandes dos Santos' ${ }^{1}$ Francisco Kelsen de Oliveira ${ }^{1,2}$, \\ Alex Sandro Gomes ${ }^{1}$, Julio José de Oliveira Ribeiro Toscano de Brito ${ }^{1}$ \\ ${ }^{1}$ Centro de Informática - Universidade Federal de Pernambuco (UFPE) Caixa \\ Postal 7851 - (81) 2126.8000 - Recife - PE - Brasil \\ ${ }^{2}$ Instituto Federal de Educação Ciência e Tecnologia do Sertão Pernambucano (IF \\ Sertão-PE) - Salgueiro, PE - Brasil \\ \{eofs,jjortb,asg\}@cin.ufpe.br, francisco.oliveira@ifsertao-pe.edu.br
}

\begin{abstract}
This article proposes to know the use and the relation of the Open Educational Resources (OER) in the processes of teaching and learning in the classroom of high schools. For this, the methodological aspects of the research took as reference the process of Systematic Review of Literature (SRL), which made it possible to identify the different works on the proposed theme. The results of this research show that in more than half of the cases of OER use there is improvement in student performance.
\end{abstract}

Resumo. Este artigo propõe conhecer o uso e a relação dos Recursos Educacionais Abertos (REA) nos processos de ensino e de aprendizagem em sala de aula de escolas do ensino médio. Para isso, os aspectos metodológicos da pesquisa tomaram como referência o processo de Revisão Sistemática da Literatura (RSL), que possibilitou identificar os diversos trabalhos sobre o tema proposto. Os resultados desta pesquisa mostram que em mais da metade dos casos de uso dos REA há melhoria no desempenho dos estudantes.

\section{Introdução}

Os Recursos Educacionais Abertos (REA), termo proveniente do inglês Open Educational Resources (OER), refere-se comumente aos objetos de ensino, aprendizagem e pesquisa disponíveis na internet sob licenças permissivas, ou seja, livres para uso, reuso, alterações e distribuições [UNESCO, 2002]. Ganhou destaque quanto ao seu potencial de uso e reuso na última década após a declaração da Cidade do Cabo sobre a Educação Aberta [Declaração da Cidade do Cabo, 2007]. Os REA podem ser encontrados na forma de planos de aula, cursos completos ou módulos, jogos, livros didáticos, artigos de pesquisa, vídeos, testes, softwares, e qualquer outra ferramenta, material ou técnica, que possa apoiar ao ensino aprendizagem [Deimann; Farrow 2013 apud Atkins et al., 2007].

Embora o termo disponha de algumas interpretações com diferentes sentidos, uma das principais propostas destes recursos é enriquecer a prática docente [Camilleri; Ehlers; Pawlowski, 2014]. Portanto, diante desta realidade houve a necessidade de 
entender como tais recursos estão sendo utilizados em sala de aula, considerando e baseando-se nos níveis "baixo" e "médio" do conceito Práticas Educacionais Abertas (PEA) analisado por Ehlers (2011), bem como a eficácia na prática docente e seu impacto no processo de aprendizagem do aluno do ensino médio, além dos possíveis fatores que influenciam o não uso desses tipos de materiais em sala de aula.

Isso foi motivado pelas lacunas de pesquisa sugeridas por Matias et al. (2016) e Oliveira, Abreu e Gomes (2015), que explicitam as necessidades de aprofundamento sobre os conhecimentos acerca das PEAs e as formas de compartilhamento de REAs e PEAs. Portanto, este trabalho teve como objetivo principal identificar e conhecer os REA utilizados em sala de aula e qual o resultado efetivo desse uso, dando respaldo para pesquisas futuras na área.

Então, a partir do método de pesquisa de revisão sistemática da literatura proposto por Kitchenham e Charters (2007), este trabalho apresenta uma conclusão baseada na resposta à uma questão de pesquisa central, sendo apoiada por quatro outras questões, sendo estas secundárias. Foi visto como resultado desta pesquisa que há melhoramento no desempenho dos estudantes em cerca de $50 \%$ dos casos de uso dos REA. Fatores como motivação do aluno e o tipo de REA utilizado estão diretamente ligados a essa conclusão.

O trabalho está dividido da seguinte forma: a próxima seção com a abordagem sobre REA e PEA, a seção seguinte com o relato do método de revisão sistemática, a penúltima seção com os resultados da pesquisa com as discussões a respeito deste resultado e a última seção com as considerações finais.

\section{Recursos Educacionais Abertos e Práticas Educacionais Abertas}

Camilleri, Ehlers e Pawlowski (2014) abordam três conceitos provenientes de diferentes fontes que servem para construir um entendimento mais generalizado sobre esse tema, embora o mais utilizado para definir REA esteja no âmbito da licença de uso. Os três conceitos trazem, de forma unânime, o fator disponibilidade, ou seja, mídias digitais disponíveis abertamente sem restrições de uso. Leva-se também em consideração seus fins não comerciais e sua utilização com o objetivo de ensinar, aprender e pesquisar.

A partir do desenvolvimento e aprimoramento dos REA, o foco em repositórios, infraestrutura e ferramentas para estas mídias aumentou e deu-se início à um momento intermediário denominado Fase 1 [Ehlers, 2011]. Segundo Rossini e Gonzalez (2012) "multiplicam-se pelo mundo os repositórios de objetos educacionais e módulos abertos", chegando a citar alguns exemplos em destaque, não se limitando a estes. Estas evidências permitem concluir que o crescimento do interesse e o incentivo à criação e adaptação dos REA direcionam o foco para um novo momento, onde serão levados em consideração tanto as políticas de produção quanto o fator pedagógico onde são utilizados.

Este novo momento é chamado de Fase 2 [Ehlers, 2011], e juntamente com esta nova discussão sobre o tema, está a questão das Práticas Educacionais Abertas (PEA). Conole e Ehlers (2010) defendem que tais práticas consistem no uso de REA com o propósito de melhorar a qualidade dos processos educacionais e inovar os ambientes 
VI Congresso Brasileiro de Informática na Educação (CBIE 2017)

Anais do XXVIII Simpósio Brasileiro de Informática na Educação (SBIE 2017)

onde acontecem esses processos. Ehlers volta a tratar do termo no ano seguinte, onde adiciona o fator políticas educacionais como meio para que a prática seja estimulada. [Ehlers, 2011].

Desta forma, entende-se que as PEA não estão limitadas às metodologias de ensino ou à uma etapa do processo de ensino-aprendizagem, no entanto também compreendem tais ações. As discussões acerca do tema tornam importantes a análise da aplicação dos REA na prática docente, levando em consideração o impacto causado pelo uso de tais recursos em sala de aula.

\section{Método de pesquisa}

Este trabalho teve como referência o processo de Revisão Sistemática da Literatura (RSL) proposto por Kitchenham e Charters (2007) e Oliveira e Gomes (2016), pois se buscou indícios relacionados ao objetivo geral desta investigação a partir dos preceitos da RSL em pesquisas relevantes sobre REA e indexadas em bases de dados científicos.

Pelo fato da revisão sistemática responder a uma pergunta claramente formulada utilizando métodos sistemáticos e explícitos para identificar, selecionar e avaliar criticamente pesquisas relevantes, além de coletar e analisar dados de estudos incluídos na revisão [Clarke, 2001], a questão de pesquisa estabelecida foi: "Qual a influência da utilização de recursos educacionais abertos na prática docente de professores do ensino médio?". A partir desta questão principal, outras quatro questões foram elaboradas com a intenção de facilitar a obtenção das respostas a partir dos trabalhos selecionados, podendo serem vistas no quadro 1:

Quadro 1. Questões de pesquisa e suas respectivas motivações.

\begin{tabular}{|c|l|l|l|}
\hline ID & QUEST ̃̃O DE PESQUISA & \multicolumn{1}{|c|}{ MOTIVAÇÃO } \\
\hline QP1 & $\begin{array}{l}\text { Quais os recursos educacionais } \\
\text { abertos que são utilizados? }\end{array}$ & $\begin{array}{l}\text { Mapear os tipos ou categorias de REA que são } \\
\text { utilizados na prática docente. }\end{array}$ \\
\hline QP2 & $\begin{array}{l}\text { Quais as disciplinas em que os } \\
\text { recursos educacionais são } \\
\text { usados? }\end{array}$ & $\begin{array}{l}\text { Mapear as disciplinas onde os REAs são } \\
\text { utilizados, e correlacionar com o tipo ou categoria } \\
\text { destes recursos. }\end{array}$ \\
\hline QP3 & $\begin{array}{l}\text { Há impacto considerável no } \\
\text { desempenho do estudante? }\end{array}$ & $\begin{array}{l}\text { Entender se o uso de REA traz alguma mudança } \\
\text { significativa no aprendizado do estudante. }\end{array}$ \\
\hline QP4 & $\begin{array}{l}\text { Qual as dificuldades } \\
\text { encontradas na prática do uso } \\
\text { dos REA? }\end{array}$ & $\begin{array}{l}\text { Considerando que haja estas dificuldades, } \\
\text { entender quais são e como estão sendo } \\
\text { trabalhadas. }\end{array}$ \\
\hline
\end{tabular}

A partir da definição das questões de pesquisa, tanto a principal quanto as secundárias, foi possível definir os termos-chave para construção da string de busca que foi utilizada na busca automática. Desta forma, os seus respectivos sinônimos também foram mapeados a fim de serem estes de igual modo utilizados. A string de busca originada a partir destes termos foi a seguinte: (teach* AND "high school" AND ("open 
VI Congresso Brasileiro de Informática na Educação (CBIE 2017)

Anais do XXVIII Simpósio Brasileiro de Informática na Educação (SBIE 2017)

educational resources" OR "OER")), ver quadro 2.

Quadro 2. Termos-chave e seus sinônimos em Inglês.

\begin{tabular}{|l|l|}
\hline \multicolumn{1}{|c|}{ Termo-chave } & \multicolumn{1}{c|}{ Sinônimo (em Inglês) } \\
\hline Prática docente & teach, teaching \\
\hline Ensino médio & high school \\
\hline REA & open educational resources, "OER" \\
\hline
\end{tabular}

Para sustentar a pesquisa automática, alguns engenhos de busca foram selecionados, sendo limitados à apenas três, e utilizados. Foram eles: IEEE Xplore, Scopus e Springer. O resultado preliminar de trabalhos retornados por cada engenho pode ser visto na seção de resultados.

A seleção dos trabalhos relevantes para a pesquisa foi possível mediante a definição dos critérios de inclusão e exclusão. Para facilitar definição dos critérios, os que serão usados para exclusão dos trabalhos estão apoiados nas três categorias a seguir: Conteúdo do trabalho, natureza do trabalho, resultado da pesquisa.

- Quanto ao conteúdo

$\circ \quad$ [CE01] - Documentos que trazem o AVA como abordagem principal

$\circ$ [CE02] - Estudos que focam no ensino superior

- [CE03] - Documentos que tratam da avaliação do uso dos REA para auto aprendizagem ou aprendizagem informal (self/informal learning)

- [CE04] - Não abordar prioritariamente o uso de REA

- Quanto ao resultado da pesquisa

$\circ$ [CE05] - Trabalhos duplicados

- Quanto a natureza do trabalho

○ [CE06] - Trabalhos a partir de 2010

$\circ$ [CE07] - Estudos incompletos

- [CE08] - Livros e capítulos de livros

Os critérios de inclusão estão listados abaixo e não foram divididos em categorias.

- [CI01] - Acesso livre do documento

- [CI02] - Estar no idioma português ou inglês

- [CI03] - Ter resultado empírico

- $\quad$ [CI04] - Ter o uso de REA em aula

A primeira etapa da seleção dos trabalhos consistiu em ler o seu título, resumo e palavras-chave. Para aumentar a eficiência do processo, mediante o número expressivo de resultados retornados, esta primeira etapa foi dividida em duas outras partes, onde a primeira [1.1] consistiu na leitura do título e palavras-chave e a segunda [1.2] a leitura do resumo.

Foram atribuídas prioridades aos critérios de inclusão e exclusão para tornar o processo de seleção mais eficiente. Os mais relevantes tinham como base acesso livre ao documento [CI01], trabalhos a partir de 2010 [CE06] e abordagem prioritária de REA [CE04]. Com isso, os trabalhos que deixassem claro apenas com a leitura do título e 
VI Congresso Brasileiro de Informática na Educação (CBIE 2017)

Anais do XXVIII Simpósio Brasileiro de Informática na Educação (SBIE 2017)

palavras-chave que os critérios primordiais não seriam atendidos, eram desconsiderados de imediato.

Alguns trabalhos não foram claros o suficiente para excluí-los ou incluí-los baseado nos critérios já citados, dessa forma adotou-se a nomenclatura "N/A" para indicar quando o trabalho não era tão claro no determinado critério. Dessa forma fez-se necessário a leitura do resumo destes trabalhos. Caso ainda não ficasse claro, o artigo passava para a etapa seguinte. A segunda etapa de seleção consistiu na leitura da introdução e conclusão e, com isso, selecionar aqueles que atendem aos critérios de inclusão e exclusão. Para auxiliar no processamento das informações, o editor de planilhas eletrônicas Google Spreadsheets foi utilizado em todas as etapas.

\section{Resultados e Discussões}

Os resultados obtidos nas buscas a partir dos engenhos de busca escolhidos identificaram 1234 estudos, sendo 968 no IEEE Xplore, 10 na Scopus e 256 na Springer. A partir destes resultados iniciais, os trabalhos retornados foram submetidos à primeira etapa do processo.

Para aumentar a sua eficiência, mediante o número expressivo de resultados retornados, esta primeira etapa foi dividida em duas partes, onde a primeira [1.1] consiste na leitura do título e palavras-chave e a segunda [1.2], a leitura do resumo. Critérios de exclusão e inclusão considerados como prioritários foram utilizados para apoiar esta etapa, sendo eles [CI01], [CE04] e [CE06]. Após a etapa 1.1, um total de 1102 trabalhos foram considerados como impróprios para a revisão, restando 132 relevantes. É importante ressaltar que há o risco de trabalhos que poderiam ser úteis terem sidos desconsiderados por não apresentarem seus títulos e palavras-chave claros o suficiente quanto aos critérios utilizados.

A etapa posterior [1.2] consistia na leitura dos resumos. Com esta etapa, a maioria dos trabalhos $(111$ - 84\%) foram claros o bastante ao fornecerem as informações necessárias para os critérios de inclusão e exclusão. Dentre estes, 7 já foram definitivamente aceitos e 104 desconsiderados para a revisão, não sendo necessário a submissão à segunda fase. No entanto os 21 restantes não foram tão claros e apenas estes passaram pela leitura da introdução e conclusão. Os critérios de inclusão e exclusão que não foram respondidos por um ou mais documentos na etapa 1.2 foram os seguintes: [CE01], [CE02], [CE03], [CE04], [CI03], [CI04]. Após serem lidos, seis trabalhos se mostraram relevantes e 15 foram desconsiderados.

Apenas 13 artigos foram considerados relevantes mediante os critérios de inclusão e exclusão e estes foram selecionados para responderem às questões de pesquisa. Nove artigos (69\%) são provenientes do IEEE Xplore, três (23\%) do Scopus e um $(8 \%)$ do Springer. O Quadro 3 mostra os trabalhos que foram selecionados.

Quadro 3. Trabalhos selecionados.

\begin{tabular}{|c|l|l|}
\hline IDENTIFICADOR & \multicolumn{1}{|c|}{ REFERÊNCIA } & BASE \\
\hline SCP07 & BISSELL(2012) & Scopus \\
\hline
\end{tabular}


VI Congresso Brasileiro de Informática na Educação (CBIE 2017)

Anais do XXVIII Simpósio Brasileiro de Informática na Educação (SBIE 2017)

\begin{tabular}{|c|l|c|}
\hline IEEE328 & DOERSCHUK (2013) & IEEE Xplore \\
\hline IEEE42 & HSU (2015) & IEEE Xplore \\
\hline SCP05 & JIMES (2013) & Scopus \\
\hline IEEE869 & KALLOO (2011) & IEEE Xplore \\
\hline IEEE447 & LAO (2010) & IEEE Xplore \\
\hline SPG92 & LEVY (2013) & Springer \\
\hline IEEE324 & MESQUITA (2013) & IEEE Xplore \\
\hline IEEE200 & PRIHATMANTO (2015) & IEEE Xplore \\
\hline IEEE435 & RUSU (2010) & IEEE Xplore \\
\hline IEEE201 & SAFITRI (2015) & IEEE Xplore \\
\hline IEEE261 & URBANO (2015) & IEEE Xplore \\
\hline SCP08 & WILEY (2012) & Scopus \\
\hline
\end{tabular}

A primeira questão [QP1] possui como propósito mapear os tipos ou categorias de REA que são utilizados na prática docente. O resultado pode ser visto no gráfico da Figura 1. A maioria dos trabalhos (10 - 77\%) trazem experiências com REA em forma de softwares. Dentre esse tipo de REA, três subcategorias se destacam: jogos (6 - 60\%), realidade aumentada $(2-20 \%)$ e simuladores $(2-20 \%)$. Além dos softwares, nos trabalhos também foram abordados livros $(2-15 \%)$ e um curso $(1-8 \%)$. Segundo o resultado dos trabalhos identificados por IEEE261, IEEE42, IEEE324, pode ser visto que os softwares estão diretamente ligados à questão da motivação dos alunos, ou seja, tornando-os mais engajados e interessados no conteúdo a ser aprendido. Além disso, os três artigos mostram que houve impacto considerável no desempenho do aluno. Isso permite entender que a motivação ou o estímulo originado pelo REA é um dos fatores que contribuem para seu uso em sala de aula.

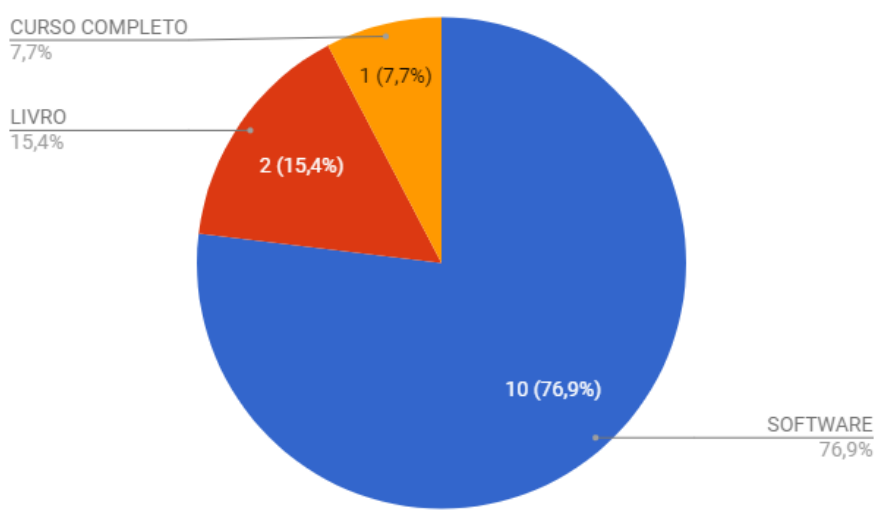

Figura 1. Resultado da Primeira questão de pesquisa

Levando em consideração o fato de softwares serem os mais utilizados nos trabalhos estudados, é interessante mostrar que este uso, no caso dos simuladores e aplicações com realidade aumentada, é viabilizado pela característica de trazer para 
VI Congresso Brasileiro de Informática na Educação (CBIE 2017)

Anais do XXVIII Simpósio Brasileiro de Informática na Educação (SBIE 2017)

mais próximo do estudante a "possibilidade de simular, manipular e visualizar processos que na prática seriam difíceis ou impossíveis de serem observáveis, assim potencializando a compreensão dos conteúdos" [Gregório; Oliveira; Matos, 2016]. Quando se trata dos jogos, de um ponto de vista psicológico, fatores como ludicidade e estimulação são essenciais para a construção do conhecimento. Câmara (2014) ainda aponta aumento significativo da motivação dos estudantes como resultado de sua pesquisa que utiliza os jogos para aumento da motivação e, consequentemente, do aprendizado.

Além de mapear os tipos de REA que são utilizados em sala de aula, este trabalho também propõe saber quais são os conteúdos em que são aplicados. Dessa forma a segunda questão de pesquisa [QP2] possui como propósito mapear as disciplinas onde os REA são utilizados, e relacionar com o tipo ou categoria destes recursos. As disciplinas onde houve a identificação de uso dos REA foram sete: Biologia, Ciências, Programação, Física, História, Matemática e Química. A relação entre tipo REA e disciplina pode ser vista no quadro 4.

Quadro 4. Mapeamento REA x Disciplina

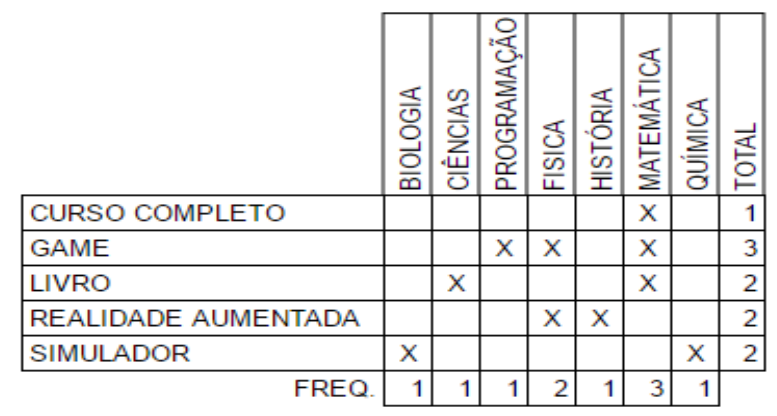

Nota-se que o game é o tipo de REA que mais se diversifica quanto a sua aplicação. Em metade dos casos essa categoria mostra-se impactante quanto ao desempenho dos alunos, o que pode ser visto nos trabalhos identificados por IEEE435, IEEE328, IEEE324. As disciplinas cujo seu uso alcança êxito no desempenho dos estudantes são Programação e Física, enquanto que na Matemática os estudantes que utilizaram estes recursos não apresentaram ganho significativo no entendimento do assunto proposto.

Seguindo com a intenção de entender qual o impacto da utilização dos REA, a terceira questão de pesquisa [QP3] propõe entender se o uso de REA traz alguma mudança significativa no aprendizado do estudante. A maioria dos trabalhos trazem resultados positivos nesse aspecto $(8-62 \%)$. Estes trabalhos utilizam, em $75 \%$ dos casos, softwares como REA. Quatro trabalhos (31\%), cada um com sua forma e metodologia de avaliação, apresentam resultados que não demonstram ganho cognitivo significativo e apenas um (7\%) não respondeu a esta questão de pesquisa, visto que este trata mais sobre a questão de usabilidade do software.

Nota-se que os dois trabalhos que abordam o uso de livros como REA possuem resultados diferentes quanto ao impacto no desempenho do aluno. Enquanto o SCP05 
mostra, baseado no relato dos professores entrevistados, que a aplicação dos livros traz um resultado satisfatório no desempenho dos alunos, SCP08 mostra que não houve resultado significativo nas avaliações que os alunos foram submetidos. Para um melhor entendimento da causa desse fato, levou-se em consideração dois pontos relevantes: a prática docente, ou seja, o uso dessa ferramenta em sala de aula sendo tradicional ou inovadora, e se o conteúdo dos livros era adaptado ao contexto sociocultural dos alunos.

Ao considerar a questão da adaptação do conteúdo, ambos os trabalhos mostram que os livros possuem conteúdos adaptados, apesar do SCP08 mostrar que essa adaptação impacta no custo do material e não no aprendizado. Quanto a prática docente, o SCP08 mostra que as práticas tradicionais são utilizadas, enquanto que o SCP05 não deixa claro como a prática acontece. Conclui-se que não há um motivo coerente para o fato do uso do mesmo tipo de recurso gerar resultados diferentes.

A última questão de pesquisa [QP4] propõe o entendimento dos fatores que podem interferir negativamente no uso dos REA, ou seja, considerando que haja dificuldades, entender quais são e como estão sendo tratadas. Apenas três artigos (23\%) relataram possíveis problemas quanto a utilização de REA. Dois deles trazem à tona a questão da adaptação do REA para o contexto a ser utilizado como um fator que dificulta o seu uso, sendo isso considerado um desafio [Jimes, Weiss, Keep. 2013], enquanto um (7\%) aborda a questão da falta de qualidade de alguns recursos como fator que pode prejudicar o aprendizado. Os demais trabalhos não respondem a esta questão.

\section{Considerações Finais}

Este estudo mapeou o estado da arte do uso de REA em sala de aula. As questões de pesquisas que foram propostas colaboram consideravelmente para o entendimento da questão principal, onde o propósito é entender qual a influência do uso dos REA na prática docente, dando foco ao fator de aprendizagem. É importante ressaltar que este estudo possui limitações que podem interferir nos seus resultados. O principal é o fato de considerar apenas três engenhos de busca de trabalhos científicos, limitando o resultado dos trabalhos que poderiam ser considerados.

Os resultados desta pesquisa mostram que em mais da metade dos casos de uso dos REA há melhoramento no desempenho dos estudantes. Fatores como motivação do aluno e o tipo de REA utilizado estão diretamente ligados a essa conclusão. Nesse sentido, os softwares estão sendo os mais utilizados, considerando os jogos, simuladores e aplicações que utilizam realidade aumentada como subcategorias. As ciências exatas, com Matemática e Física sendo citadas, estão no topo das disciplinas que mais utilizam REA, sendo os jogos os mais inseridos neste contexto. Como dificuldades encontradas na prática com estes recursos estão as questões da adaptação do conteúdo e a falta de qualidade de alguns deles.

\section{Referências}

Bissell (2012). Architecture and Impact of an Open, Online, Remixable, and Multimedia-Rich Algebra 1 Course. Journal of asynchronous learning networks, 
VI Congresso Brasileiro de Informática na Educação (CBIE 2017)

Anais do XXVIII Simpósio Brasileiro de Informática na Educação (SBIE 2017)

$16(5), 49-59$.

Câmara, B. B. A. (2014). Motivação e games: o uso do jogo Angry Birds com estudantes para o ensino de física.

Camilleri, A. F., Ehlers, U. D., \& Pawlowski, J. (2014). "State of the art review of quality issues related to open educational resources (OER)." Luxembourg: Publications Office of the European Union.

Clarke, M. O. 2001. "Formulating the problem". Cochrane Reviewers' Handbook. England: The Cochrane Collaboration. Disponível em: http://www.cochrane.dk/cochrane/handbook/handbook.htm. Acesso em: 11 jun. 2017.

Conole, G.C.; Ehlers, U.D. (2010). “Open Educational Practices: Unleashing the power of OER.” Paper presented to UNESCO Workshop on OER in Namibia 2010.

Declaração da cidade do cabo. (2007) "Declaração de Cidade do Cabo para Educação Aberta: Abrindo a promessa de Recursos Educativos Abertos". Cape Town. Disponível em: http://www.capetowndeclaration.org/translations/portuguesetranslation. Acesso em: 21 abr. 2017.

Deimann, M.; Farrow, R. (2013) "Rethinking OER and their use: Open education as bildung". International Review of Research in Open and Distance Learning, v. 14, n. 3, p. 344-360.

Doerschuk (2013). Introducing programming concepts through video game creation. In Frontiers in Education Conference, 2013 IEEE (pp. 523-529). IEEE.

Ehlers, Ulf-daniel. (2011). "From open educational resources to open educational practices”. Elearning Papers 23.

Gregório, E. A., de Oliveira, L. G., \& de Matos, S. A. "Uso de simuladores somo ferramenta no ensino de conceitos abstratos de Biologia: Uma proposição investigativa para o ensino de síntese proteica." Experiências em Ensino de Ciências V.11, No. 1, 2016.

Hsu (2015). Teaching High School Computer Science with Videos of Historical Figures--An Augmented Reality Approach. In Learning and Teaching in Computing and Engineering (LaTiCE), 2015 International Conference on (pp. 22-25). IEEE.

Jimes (2013). Addressing the Local in Localization: A Case Study of Open Textbook Adoption by Three South African Teachers. Journal of Asynchronous Learning Networks, 17(2), 73-86.

Jimes, C., Weiss, S., \& Keep, R. (2013). "Addressing the Local in Localization: A Case Study of Open Textbook Adoption by Three South African Teachers." Journal of Asynchronous Learning Networks, 17(2), 73-86.

Kalloo (2011). Correlation between student performance and use of an mLearning application for high school mathematics. In Advanced Learning Technologies (ICALT), 2011 11th IEEE International Conference on (pp. 174-178). IEEE.

Kitchenham, B. A. Charters, S. Guidelines for performing Systematic Literature 
VI Congresso Brasileiro de Informática na Educação (CBIE 2017)

Anais do XXVIII Simpósio Brasileiro de Informática na Educação (SBIE 2017)

Reviews in Software Engineering. Technical Report EBSE-2007-01, 2007.

Lao (2010). Developing a Computer-Based Learning Tool for Biology. In Embedded and Multimedia Computing (EMC), 2010 5th International Conference on (pp. 1-6). IEEE.

Levy (2013). How dynamic visualization technology can support molecular reasoning. Journal of Science Education and Technology, 1-16.

Matias, P. H. B. et al. (2016) "Ambiente de compartilhamento de REAs: uma proposta de modelagem, implementação e modelo de negócio". Revista Semiárido De Visu, v. 4, n. 1, p. 18-31. Disponível em: http://semiaridodevisu.ifsertaope.edu.br/index.php/revista/article/view/195/149. Acesso em: 29 abr. 2017.

Mesquita (2013). Education for energy efficiency through an educational game. In Frontiers in Education Conference, 2013 IEEE (pp. 535-540). IEEE.

Oliveira, F. K.; Gomes, A. S. (2016). "Revisão Sistemática da Literatura". In: Oliveira, F. K.; Abreu, K. F. (Eds.). "Métodos e pesquisas em Educação". 1. ed. Brasília: Editora Kiron. p. 164.

Oliveira, F. K.; Abreu, K. F.; Gomes, A. A. S. "Formação profissional em recursos educacionais abertos". Revista Semiárido De Visu, v. 3, n. 2, p. 98-109. Disponível em: http://semiaridodevisu.ifsertao-pe.edu.br/index.php/revista/article/view/205/132. Acesso em: 29 abr. 2017.

Prihatmanto (2015). Educational game design calculation of broad and round the triangle and quadrilateral using DPE (Design, play, and experience) framework. In Interactive Digital Media (ICIDM), 2015 4th International Conference on (pp. 1-6). IEEE.

Rossini, C.; Gonzalez, C. (2012) "O debate em política pública e as oportunidades para o mercado". In: Santana, B.; Rossini, C.; Pretto, N. L. (Org.). Recursos Educacionais Abertos: práticas colaborativas políticas públicas. Salvador: EDUFBA.

Rusu (2010). Learning software engineering basic concepts using a five-phase game. In Frontiers in Education Conference (FIE), 2010 IEEE (pp. S2D-1). IEEE.

Safitri (2015). Design and implementation of educational game based on thematic curriculum using three layered thinking model (Case study: Applying number and social arithmetic in the real life). In Interactive Digital Media (ICIDM), 2015 4th International Conference on (pp. 1-7). IEEE.

UNESCO (2002) "Forum on the Impact of Open Courseware for Higher Education in Developing Countries funded by the Hewlett Foundation".

Urbano (2015). How Students and Teachers React to an AR free Puzzle Game: preliminary tests. In Global Engineering Education Conference (EDUCON), 2015 IEEE (pp. 852-855). IEEE.

Wiley (2012). A preliminary examination of the cost savings and learning impacts of using open textbooks in middle and high school science classes. The International Review Of Research In Open And Distributed Learning, 13(3), 262-276. 